\title{
Improvement of survival with postmastectomy radiotherapy in patients with 1-3 positive axillary lymph nodes: A systematic review and meta-analysis of the current literature
}

\author{
HANNAH HEADON, ABDUL KASEM, REHAM ALMUKBEL and KEFAH MOKBEL
}

The London Breast Institute, The Princess Grace Hospital, W1U 5NY London, UK

Received December 14, 2015; Accepted March 21, 2016

DOI: $10.3892 / \mathrm{mco} .2016 .971$

\begin{abstract}
In breast cancer with $>4$ positive axillary lymph nodes, it is common practice to deliver radiotherapy to the affected site following mastectomy. However, less is known regarding the benefits this may confer on women with 1-3 positive lymph nodes. In this meta-analysis, we aimed to assess whether post-mastectomy radiotherapy (PMRT) was beneficial for such patients. A literature review was conducted using the PubMed and Ovid databases. Selected studies were analysed and data regarding overall survival (OS) and locoregional recurrence (LRR) rates were extracted. Statistical analysis was then conducted in order to develop a combined risk ratio (RR) for both OS and LRR in the setting of PMRT in women with breast cancer with 1-3 positive lymph nodes. PMRT in women with 1-3 positive lymph nodes significantly reduced the risk of LRR, with a RR of 0.3 [95\% confidence interval (CI): $0.23-0.38]$ and also showed a minor benefit in terms of OS (RR=1.03, 95\% CI: 1.00-1.07). Therefore, in breast cancer patients with 1-3 positive lymph nodes, PMRT significantly reduced the risk of LRR and was associated with a minor OS benefit. Until the results of ongoing randomised controlled trials are published, PMRT should be recommended in this group of patients following a careful multidisciplinary discussion.
\end{abstract}

\section{Introduction}

In breast cancer, a common treatment for achieving local control is for the patient to undergo mastectomy in order to remove any detectable macroscopic disease. In early-stage disease, this aims to remove the tumour and, therefore, reduce the incidence of metastasis. However, mastectomy is not always able to remove all disease foci, which may remain in the

Correspondence to: Professor Kefah Mokbel, The London Breast Institute, The Princess Grace Hospital, 42-52 Nottingham Place, W1U 5NY London, UK

E-mail: kefahmokbel@hotmail.com

Key words: postmastectomy radiotherapy, axillary lymph nodes, breast cancer locoregional tissue. This may lead to locoregional recurrence (LRR) and, subsequently, in some cases, death from breast cancer. Radiotherapy, when used as an adjuvant therapy, has the potential to remove small disease foci, thereby reducing the risk of LRR.

The use of post-mastectomy radiotherapy (PMRT) has long been established in the treatment of patients with T3/4 breast cancer and/or those with $\geq 4$ positive axillary lymph nodes, having been associated with a clear survival benefit and reduction in local recurrence, evidence that has reached a level of 1a (1). Therefore, its use in such patients is currently recommended by several national bodies, including the National Institute for Health and Clinical Excellence (2). The central issue currently is the role of PMRT in intermediate-risk patients, meaning those with 1-3 positive lymph nodes. A meta-analysis published in 2014 demonstrated that the beneficial effects of PMRT remained apparent in such patients, who received the same benefit as those with more positive nodes (3), although no additional benefit was observed in those without positive nodes.

The aim of the present meta-analysis was to build upon the evidence presented previously by focusing on the effect of PMRT on overall survival (OS) and LRR in patients with 1-3 positive axillary lymph nodes, regardless of the use of systemic therapy, by including data from more recent studies.

\section{Materials and methods}

Types of studies and participants. Prospective clinical trials and retrospective case series with reported outcomes as a function of PMRT in breast cancer patients with 1-3 positive axillary lymph nodes were considered. All the selected studies included female adult patients with primary breast cancer and positive metastases to 1-3 axillary lymph nodes, and all the patients were treated with mastectomy, with or without PMRT.

Outcome measures. The primary outcome was OS in patients treated with PMRT in the setting of primary breast cancer. The secondary endpoint was LRR, when reported.

Search methods. A computer-aided search through the PubMed and Ovid databases was performed to identify relevant literature. The lower limit date for the search was set at 01/04/2015, with no upper limit. The following search terms were used: 'post-mastectomy radiotherapy 1-3 lymph nodes 
survival', 'post-mastectomy 1-3 lymph nodes', 'radiotherapy post-mastectomy <3 lymph nodes' and 'post-mastectomy'. The related articles function on PubMed was also utilised and the bibliographies of relevant articles were analysed in order to identify all relevant literature.

Data collection and analysis. The authors independently performed the study selection according to the inclusion criteria outlined above. Studies in full text were selected if they reported: i) Either OS, or LRR, or both, for adult female breast cancer patients who were treated with mastectomy and PMRT compared with patients undergoing mastectomy without PMRT in the presence of 1-3 positive axillary lymph nodes; and ii) full text was available for data extraction. The exclusion criteria were: i) Studies that did not report OS or LRR; and ii) case reports, commentaries, letters or reviews.

Data extraction. The authors extracted data independently using the following items: Characteristics of included studies (author, publication date, study design, participants and interventions), median age of the participants and the aforementioned outcomes.

Measure of treatment effect and statistical analysis. Percentages and their $95 \%$ confidence intervals (CIs) for OS and/or LRR as a function of the use of PMRT in patients with 1-3 positive lymph nodes were retrieved from each included study. A meta-analysis of each outcome was then performed, following assessment for heterogeneity using Cochrane's Q and $\mathrm{I}^{2}$ tests. The results of these tests, plus a zero-effect test, determined the use of either a fixed-effects or random-effects model. Potential publication biases were evaluated with funnel plots for OS and LRR in order to examine the relative symmetry of individual study estimates around the overall estimate in addition to Duval and Tweedie's trim and fill method. This was accompanied by Begg's and Egger's tests. $\mathrm{P}<0.05$ was considered to indicate statistically significant differences.

The results were reported as a classic forest plot, one for each outcome of OS and LRR. All the statistical analyses were performed using RevMan 5.1 and Comprehensive Meta-Analysis, version 2 software (Comprehensive MetaAnalysis Software, Englewood NJ, USA).

\section{Results}

A total of 943 publications were identified, 14 of which were included in this review (Tables I and IV), incorporating a total of 8,544 patients. The flow diagram of the study selection process is shown in Fig. 1. A total of 13 studies were excluded, as they did not include reports of either OS or LRR rates as part of their results. All included studies were retrospective case series. The primary endpoints of either OS or LRR rate, along with 95\% CIs were reported, or could be calculated for all the studies included. The pooled relative risk ratio (RR) for OS was 1.03 (95\% CI: 1.00-1.07) and for LRR it was 0.30 (95\% CI: 0.23-0.38), showing a benefit in delivering PMRT to patients with 1-3 positive lymph nodes.

OS. For OS, a total of 9 studies were included (Table I), incorporating 5,837 patients with a mean follow-up of 80.4 months

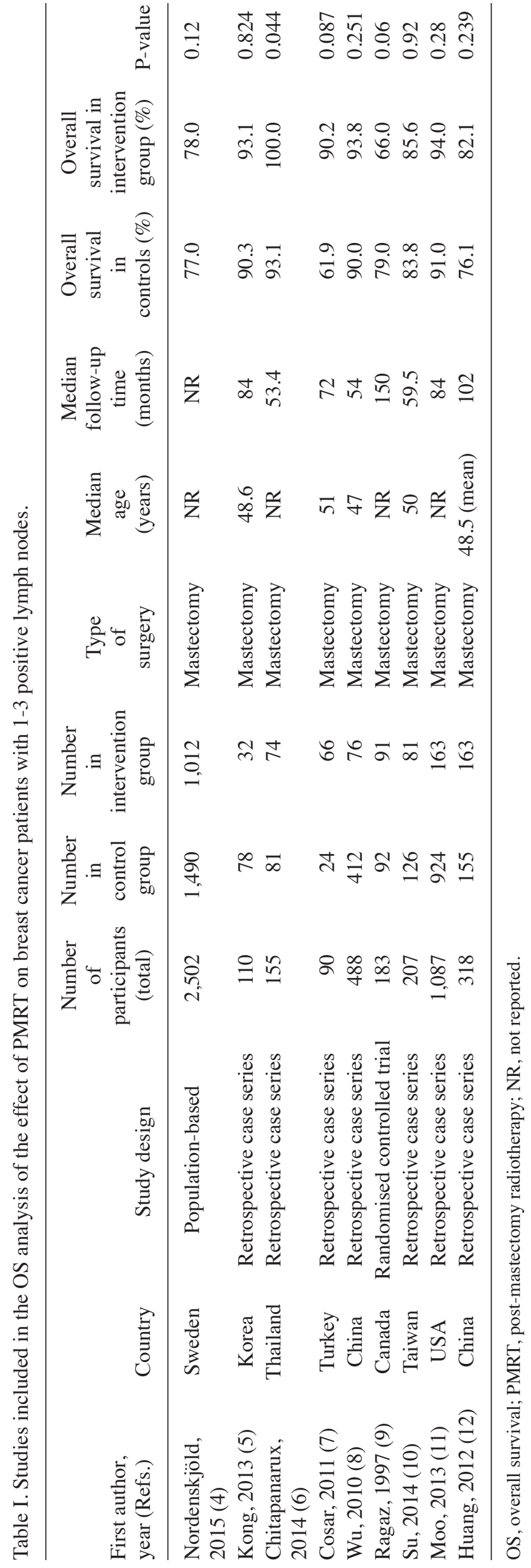


Table II. Heterogeneity tests for overall survival.

\begin{tabular}{|c|c|c|c|c|c|}
\hline Test $^{\mathrm{a}}$ & Null vs. alternative/thresholds & Measure & Df & $\chi^{2}$ & Prob level \\
\hline Cochran's Q & $\begin{array}{l}H_{0}: \text { All studies are evaluating the same effect } \\
H_{a}: \text { Not all studies are evaluating the same effect }\end{array}$ & Risk ratio & 8 & $\chi^{2}=13.7$ & 0.089928 \\
\hline $\mathrm{I}^{2}$ & $\begin{array}{l}0 \text { to } 40 \% \text { : May not be important } \\
30 \text { to } 60 \% \text { : May represent moderate heterogeneity } \\
50 \text { to } 90 \% \text { : May represent substantial heterogeneity } \\
75 \text { to } 100 \% \text { : Indicates considerable heterogeneity }\end{array}$ & Risk ratio & & $\mathrm{I}^{2}=42$ & \\
\hline
\end{tabular}

${ }^{a}$ Tests performed with RevMan. Cochran's Q and $\mathrm{I}^{2}$ tests demonstrated that the null hypothesis may be rejected at $5 \%$ level of significance and that the included studies exhibited moderate heterogeneity. Df, degree of freedom.

(range, 53.4-150 months). Information collected included total participants in the treatment and control arms and respective OS rates. The mean follow-up time was 80.4 months. First, heterogeneity was assessed according to Cochran's Q and $\mathrm{I}^{2}$ tests. Cochran's Q test suggested that the null hypothesis (that the treatment effect would be equal to 0 ) could be rejected, whilst the $\mathrm{I}^{2}$ was calculated at $42 \%$, indicating moderate heterogeneity (Table II). To account for this heterogeneity, we calculated summary statistics using the random-effects model.

RRs were calculated from the results of the studies listed in Fig. 2. The summary RR was then calculated as 1.03 (95\% CI: 1.00-1.07). Therefore, according to the summary effect, OS is $3 \%$ higher following PMRT. When the relative risk measure value is equal to 1.00 , it indicates no difference in OS between intervention and control groups. As the lower limit of the $95 \%$ CI is 1.00 , an additional zero-effect test was performed, based on the natural logarithms of RRs (Table III).

Both tests achieved statistical significance (set at $\mathrm{P}<0.05$ ) at the 5\% significance level; therefore, the null hypothesis can be rejected. Furthermore, we may conclude that PMRT exerts a small but positive effect on the OS of patients.

The last item in our analysis was to estimate the publication bias of the included studies by incorporating them into a funnel plot. Within the funnel plot, not all studies are within the $95 \% \mathrm{CI}$, and it is not conclusive whether all the studies are symmetrical around the combined effect size, indicating absence of publication bias. Using Duval and Tweedie's trim and fill method imputes an allegedly omitted study with a natural logarithm RR of 0.35 . The recomputed combined effect estimate remains very close to our initial estimate of 1.03 on the random-effects model: 1.03 (95\% CI: 0.99-1.07) vs. 1.03 (95\% CI: 1.00-1.07), respectively. The Begg and Mazumdar rank correlation test also supported the absence of publication bias, showing no correlation between the study size and the effect size. In conclusion, we may support our estimate of a summary RR=1.03, with a 95\% CI of 1.00-1.07.

$L R R$. The effect of PMRT on LRR was also analysed using 11 studies (5,7,9-17), incorporating 5,399 patients (Fig. 3, Table IV). The mean follow-up time was calculated as 91.2 months (range, 59.5-150 months). The effect of the intervention was again estimated using the RR measure. We used the zero-effect test, Cochrane's Q and $\mathrm{I}^{2}$ tests for heterogeneity, summary effect using a forest plot, and checked for publication

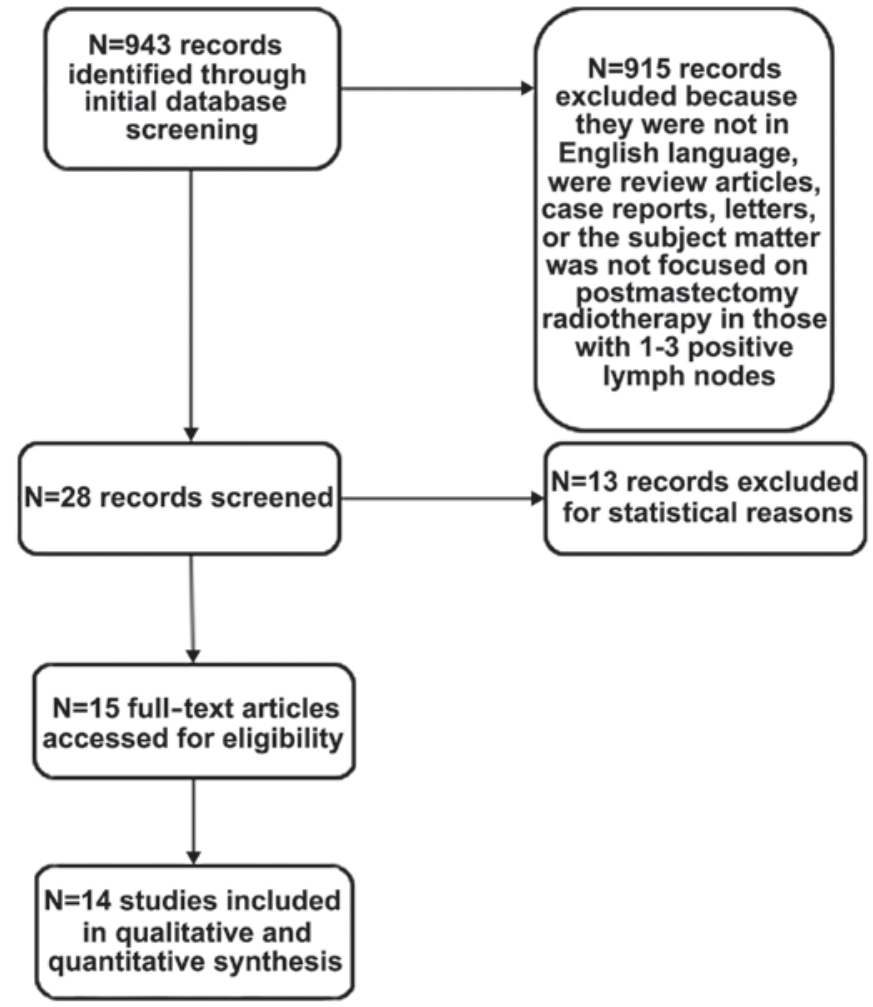

Figure 1. Flow chart outlining the methodology followed through the process of the literature review.

bias using a funnel plot, Egger's test of the intercept, and Begg and Mazumdar rank correlation test.

Using the zero-effect test, we were able to reject the null hypothesis, in which the effect is equal to 0 , corresponding to a RR of 1 . Hence, it may be predicted that PMRT should exert a statistically significant effect on LRR (Table V).

Cochran's $\mathrm{Q}$ and $\mathrm{I}^{2}$ tests suggest that all the studies are evaluating the same effect, and heterogeneity is not significantly present (Table VI). Therefore, the fixed-effects model may be used to estimate the combined effect.

The summary effect was calculated using a fixed-effects model, which was incorporated into a forest plot (Fig. 3). The combined RR of the effect of PMRT on LRR was calculated as 0.30 (95\% CI: 0.23-0.38), indicating that PMRT considerably decreases the risk of LRR. 
Table III. Zero-effect tests.

\begin{tabular}{|c|c|c|c|c|c|}
\hline Test $^{\mathrm{a}}$ & Null vs. alternative & Measure & Df & $\chi^{2}$ & Prob level \\
\hline Non-directional & $\begin{array}{l}H_{0}: \text { all treatment effects are zero } \\
H_{a}: \text { at least one is not zero }\end{array}$ & $\ln (\mathrm{RR})$ & 9 & 20.06 & 0.017546 \\
\hline Directional & $\begin{array}{l}H_{0}: \text { all treatment effects are zero } \\
H_{a}: \text { effects are equal to the same non-zero quantity }\end{array}$ & $\ln (\mathrm{RR})$ & 1 & 6.86 & 0.008815 \\
\hline
\end{tabular}

${ }^{\mathrm{a}}$ Manual calculation of test statistics based on $\ln (\mathrm{RR})$ and SE $\ln (\mathrm{RR})$ output from Comprehensive Meta-Analysis, version 2.0. Both tests show significance at the 5\% significance level, so the null hypothesis may be rejected. Df, degree of freedom; RR, risk ratio; SE, standard error.

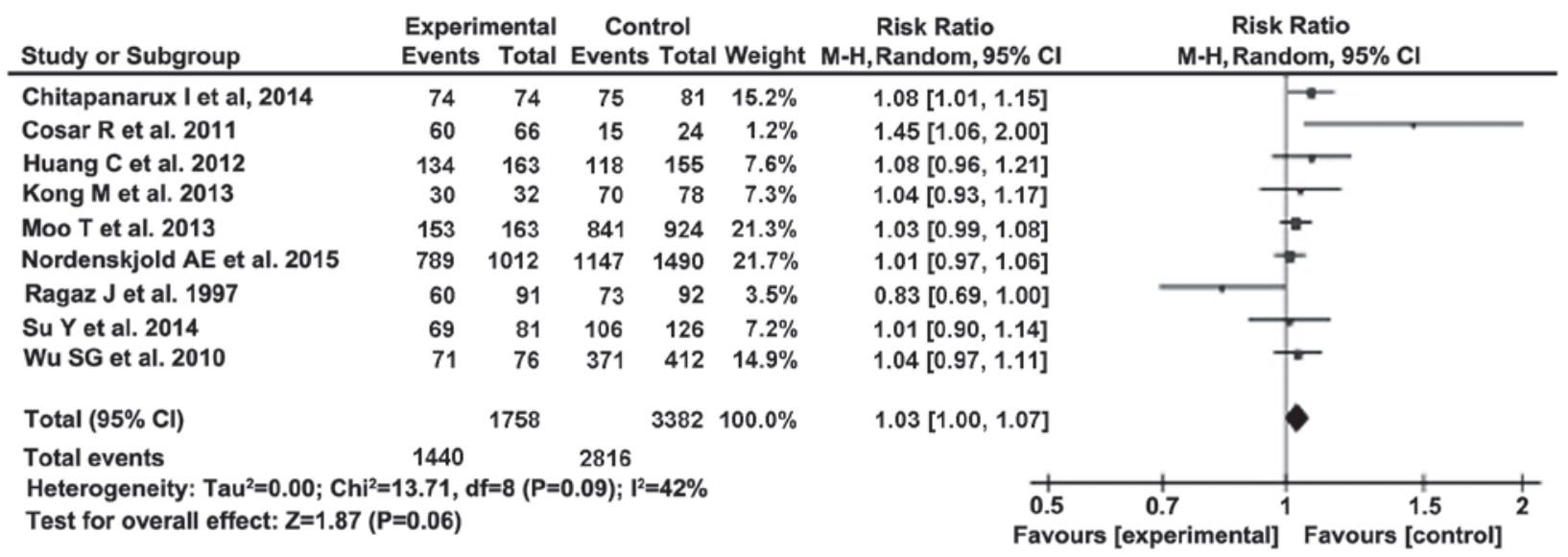

Figure 2. Forest plot showing the summary relative risk ratio of overall survival in breast cancer patients with 1-3 positive axillary lymph nodes undergoing post-mastectomy radiotherapy. This forest plot outlines the studies included in the overall survival analysis and demonstrates the summary relative risk ratio, which was calculated at 1.03 , therefore favouring the intervention. CI, confidence interval.

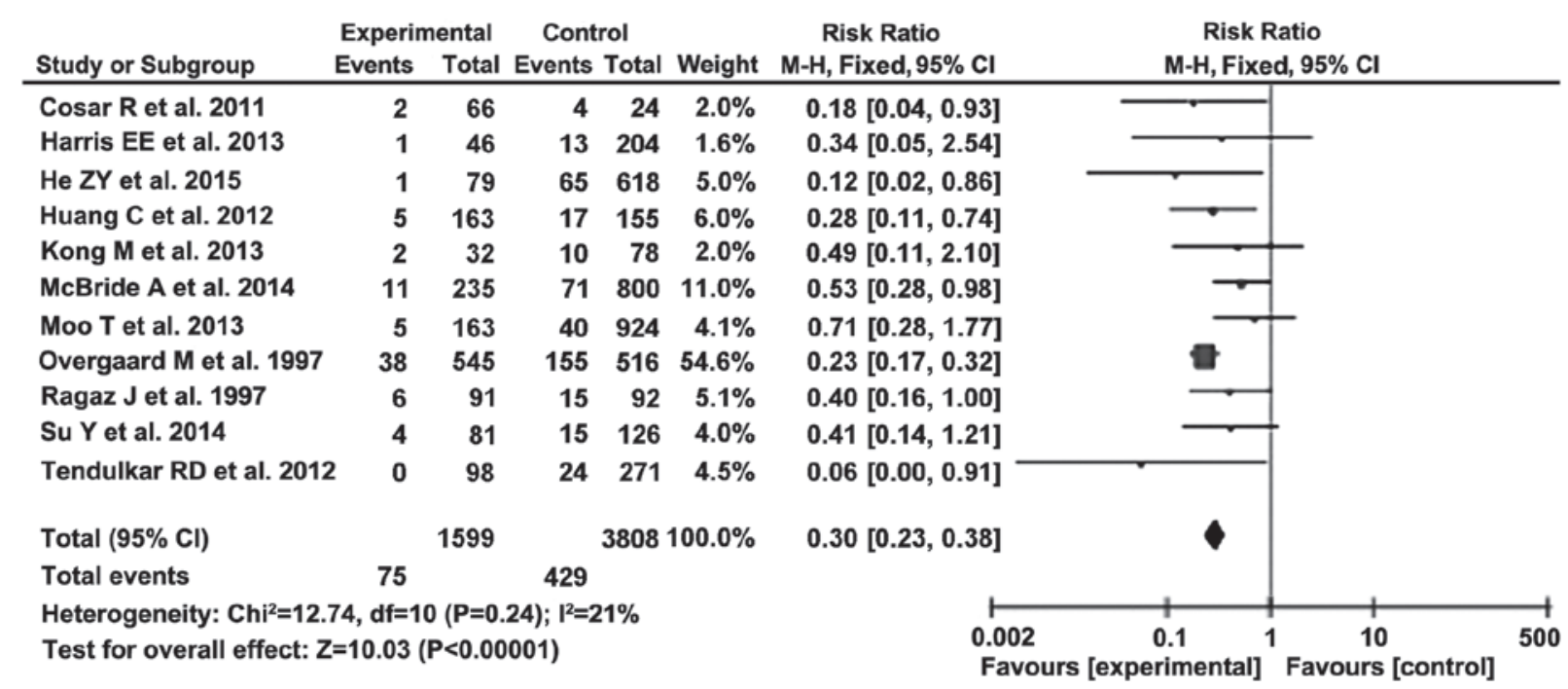

Figure 3. Forest plot showing the summary relative risk ratio of locoregional recurrence in breast cancer patients with $1-3$ positive axillary lymph nodes undergoing post-mastectomy radiotherapy. This forest plot outlines the studies included in the locoregional recurrence analysis and demonstrates a summary relative risk ratio of 0.30 , therefore favouring the intervention by indicating that post-mastectomy radiotherapy reduces the risk of locoregional recurrence in these patients. CI, confidence interval.

To check for publication bias, a funnel plot was created using Duval and Tweedie's trim and fill method. Additionally, Egger's test and Begg and Mazumdar rank correlation test were performed. On the funnel plot, all estimates were within the $95 \% \mathrm{CI}$ and were placed relatively symmetrically around the combined effect, indicating no publication bias. Duval and Tweedie's trim and fill method did not signify any missing study, generating an unchanged estimate of the combined RR. The results of the Egger's test and Begg and Mazumdar tests are outlined in Table VII. These concluded that there was no 


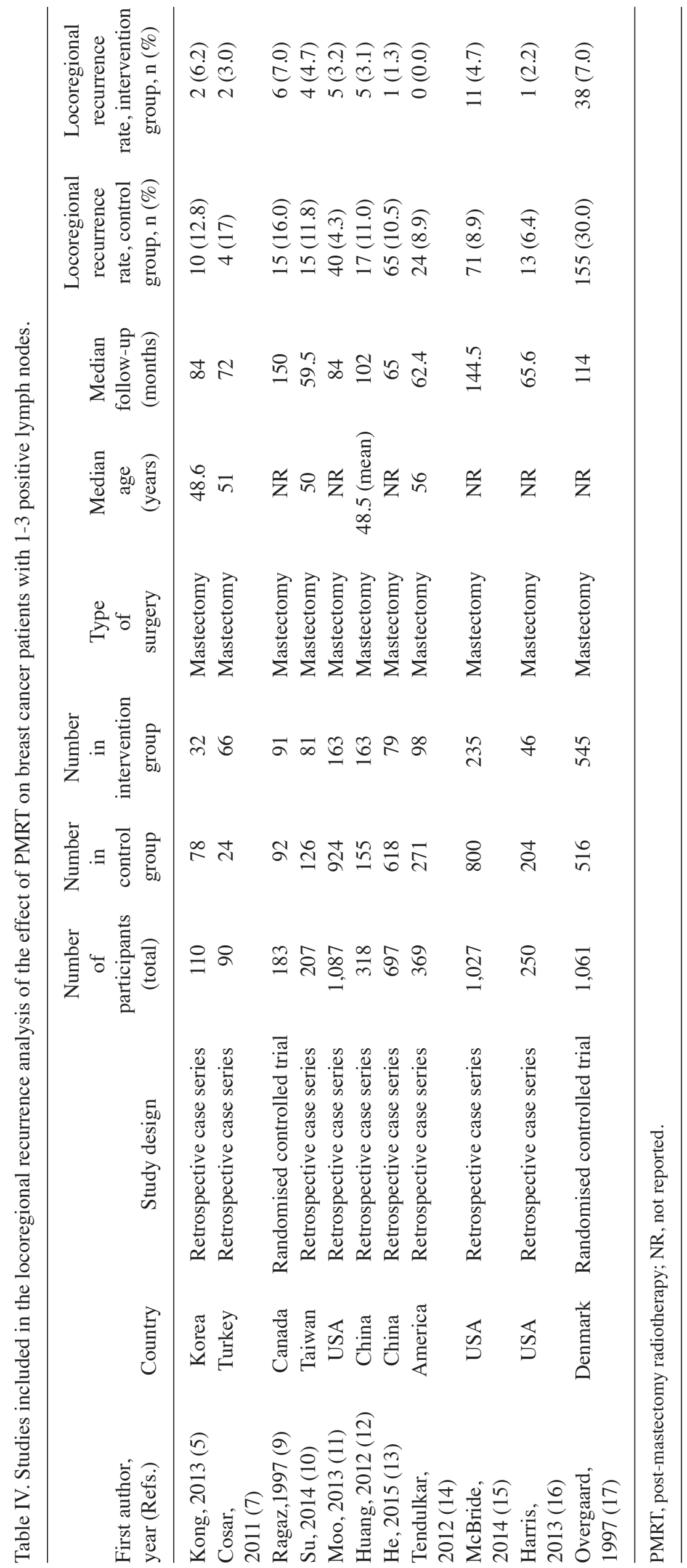


Table V. Zero-effect test rejecting the null hypothesis.

\begin{tabular}{|c|c|c|c|c|c|}
\hline Test $^{\mathrm{a}}$ & Null vs. alternative & Measure & Df & $\chi^{2}$ & Prob level \\
\hline Non-directional & $\begin{array}{l}H_{0}: \text { all treatment effects are zero } \\
H_{a}: \text { at least one is not zero }\end{array}$ & $\ln (\mathrm{RR})$ & 11 & 106.11 & 0.0000 \\
\hline Directional & $\begin{array}{l}H_{0}: \text { all treatment effects are zero } \\
H_{a}: \text { effects are equal to the same non-zero quantity }\end{array}$ & $\ln (\mathrm{RR})$ & 1 & 93.46 & 0.0000 \\
\hline
\end{tabular}

${ }^{\mathrm{a}}$ Manual calculation of test statistics based on $\ln (\mathrm{RR})$ and SE $\ln (\mathrm{RR})$ output from Comprehensive Meta-Analysis, version 2.0. Df, degree of freedom; RR, risk ratio; SE, standard error.

Table VI. Tests of heterogeneity showing non-significant heterogeneity and that all studies are evaluating the same effect (fixed-effects model).

\begin{tabular}{|c|c|c|c|c|c|}
\hline Test $^{\mathrm{a}}$ & Null vs. alternative/thresholds & Measure & Df & $\chi^{2}$ & Prob level \\
\hline Cochran's Q & $\begin{array}{l}H_{0}: \text { all studies are evaluating the same effect } \\
H_{a}: \text { not all studies are evaluating the same effect }\end{array}$ & Risk ratio & 10 & $\chi^{2}=12.7$ & 0.240932 \\
\hline $\mathrm{I}^{2}$ & $\begin{array}{l}0 \text { to } 40 \% \text { : May not be important } \\
30 \text { to } 60 \% \text { : May represent moderate heterogeneity } \\
50 \text { to } 90 \% \text { : May represent substantial heterogeneity } \\
75 \text { to } 100 \% \text { : Indicates considerable heterogeneity }\end{array}$ & Risk ratio & & $\mathrm{I}^{2}=21 \%$ & \\
\hline
\end{tabular}

${ }^{a}$ Tests performed in RevMan. Df, degree of freedom.

Table VII. Tests for publication bias indicating that there was no sign of any missing study and, therefore, the estimate of the combined risk ratio remained unchanged (no publication bias).

\begin{tabular}{ll}
\hline Test $^{\mathrm{a}}$ & \multicolumn{1}{c}{ Results } \\
\hline Egger's test of the intercept & $\begin{array}{l}\text { Intercept }(\mathrm{B} 0) \text { is } 0.22971,95 \% \text { confidence interval }(-1.19571,1.65513), \\
\text { with } \mathrm{t}=0.36455, \text { degree of freedom }=9 \text {. The 1-tailed P-value (recommended) } \\
\text { is } 0.36193, \text { and the 2-tailed P-value is } 0.72387\end{array}$ \\
$\begin{array}{l}\text { Kendall's tau b (corrected for ties, if any) is }-0.32727, \text { with a 1-tailed P-value } \\
\text { (recommended) of } 0.08056 \text { or a 2-tailed P-value of } 0.16112 \text { (based on } \\
\text { correlation test } \\
\text { continuity-corrected normal approximation) }\end{array}$
\end{tabular}

${ }^{a}$ Analysis performed with Comprehensive Meta-Analysis, version 2.

publication bias. Therefore, we may support the estimate of a summary RR of 0.30 (95\% CI: 0.23-0.38), indicating that PMRT significantly reduces the risk of LRR in breast cancer.

Overall results of the meta-analysis. Overall, the results of the meta-analysis demonstrated that PMRT significantly decreased the risk of LRR $(R R=0.30,95 \%$ CI: $0.23-0.38)$, whereas there was a non-significant increase in $\mathrm{OS}(\mathrm{RR}=1.03$, 95\% CI: $1.00-1.07)$.

\section{Discussion}

The meta-analysis results demonstrated that PMRT appears to significantly reduce the risk of LRR, with a minor benefit in terms of OS. These results, therefore, support the use of PMRT to reduce LRR in breast cancer patients, with a small chance of increasing OS. The small, non-significant benefit in OS through using PMRT may be explained by the follow-up times used in the studies analysed. The mean follow-up time was 80.4 months (range, 53.4-150 months). A longer follow-up time may allow the significant benefit seen in LRR to translate to an increased benefit in OS. Additionally, a number of other risk factors, which were not included in the present study, may also affect these outcomes. Tumour factors, such as receptor status and size, have also been found to affect both LRR and OS. Other information, which may exert an effect on OS, includes comorbidities and patient age.

In this meta-analysis, we did not control for any additional factors that may also have an impact on OS and LRR rates. However, some of the studies that were included performed multivariate analyses in order to identify any independent prognostic factors. In terms of OS, younger age, medial tumour 
location, HER2/neu overexpression and negative oestrogen receptor status were associated with poorer outcomes and a reduction in OS $(4-6,10,12)$. Higher-grade disease, a triple-negative subtype, age $<40$ years, HER2/neu overexpression and negative oestrogen receptor status were all identified as independent poor prognostic factors for $\operatorname{LRR}(5,6,10,12,13)$. In order to fully elucidate the effect of these factors, and other factors, such as the use of systemic therapy, on the suggested LRR rates and OS when PMRT is used, future prospective randomised trials are warranted. This may enable the identification of a subgroup of patients for whom PMRT may be particularly beneficial, in terms of reducing LRR and increasing OS.

The Early Breast Cancer Trialists' Collaborative Group recently conducted a meta-analysis investigating the effect of PMRT on 10-year recurrence and 20-year breast cancer mortality. Although not reporting specifically on women with only 1-3 positive lymph nodes, they did report on the effect of PMRT on LRR within this group, and demonstrated that PMRT significantly reduced the risk of LRR, as well as overall recurrence and breast cancer mortality (3). However, due to the long follow-up used in this trial, the patients included were treated a long time ago; therefore, systemic therapy, which was used additionally, would not have been as effective as the systemic therapy currently used. Therefore, the benefits exclusively from PMRT in modern trials are likely to be smaller, due to the use of modern systemic therapy, such as Herceptin, endocrine therapy and improved chemotherapy regimens.

One of the main issues with PMRT is the risk of cardiac toxicity caused by chest wall irradiation. Cardiac irradiation has been associated with significant pathological damage to the heart, such as microcirculatory damage, which may lead to ischaemia, fibrosis, accelerated atherosclerosis, pericardial effusion and pericardial thickening (18). Earlier studies associated PMRT with adverse cardiac effects, such as myocardial infarctions, and a significantly increased number of cardiac deaths, with the left anterior descending coronary artery suggested to be particularly vulnerable to damage (19). Despite this, more recent studies have dispelled these findings, with one prospective trial showing no clinically significant acute or late cardiac adverse events at the 2-year follow-up, and no difference in left ventricular ejection fraction (18). Although this particular trial had a short follow-up time, the reduction in the risk of adverse cardiac events has been attributed to the advancements in radiotherapy techniques. For example, the use of three-dimensional computed tomography-guided planning in order to minimise the exposure of the heart has reduced the effects of late cardiac toxicity $(19,20)$. In a study by Doyle et al, it was reported that the use of radiotherapy did not increase the risk of myocardial infarction over a period of 10 years (21). Subsequently, it may be concluded that, with proper planning, PMRT does not necessarily increase the risk of cardiac adverse effects, although it would be useful to conduct a trial assessing this risk in patients with 1-3 positive lymph nodes, in order to fully establish whether the benefits regarding LRR incidence outweigh any risks to the heart.

In addition, the adverse effects of PMRT on breast reconstruction should be considered in the benefit-risk analysis in the context of LRR incidence and OS benefits. PMRT increases the complication rate of any type of reconstruction, autologous or implant-based. Most guidelines also suggest it is better to delay reconstruction if it is known preoperatively that radiotherapy will be required. However, immediate reconstruction is associated with a better quality of life and reduces the risks of undergoing a second surgery (22). Despite this, it has been suggested that radiotherapy performed after reconstruction may lead to a higher complication rate than if reconstruction is delayed. In a previous study investigating the use of adjuvant radiotherapy in porcine acellular dermis-assisted breast reconstruction, the rate of total complications and implant/expander loss was significantly higher in irradiated breasts (23). Likewise, a study investigating immediate autologous reconstruction found that there was an increased risk of fat necrosis when the breasts were irradiated (24). Therefore, it is important to take this into account when planning the care of patients who are likely to require radiotherapy, in order to minimise the complications and optimise the aesthetic outcome.

There were a number of limitations to this meta-analysis. All the studies included were retrospective case series, whereas, ideally, prospective randomised trials would be useful in order to increase the reliability of the results. Additionally, a relatively limited number of studies were included in each section. Despite this, many of the studies were published in the last 5 years, indicating that this is a growing area of research; therefore, future analyses may be able to draw their conclusions from a significantly larger pool of research. For example, the SUPREMO trial in the UK, which is currently being undertaken, aims to determine the effect of PMRT on OS in women at intermediate risk of LRR. However, the results will not be available for a number of years, due to a minimal 10-year follow-up (25).

In conclusion, PMRT in women with breast cancer with 1-3 positive lymph nodes results is associated with a significant decrease in LRR and a relatively small OS benefit. In view of the fact that the OS benefit is relatively small at $3 \%$, it would be reasonable to recommend PMRT to a selected group of patients with other risk factors, such as young age, oestrogen receptor-negative, HER2-positive, large, poorly differentiated tumours, following detailed multidisciplinary discussion until the results of ongoing, large-scale randomised controlled trials become known. In light of the risk of cardiac toxicity, the threshold for recommending PMRT will be lower for tumours of the right breast, where there is a lower risk of adverse cardiac effects. The results of this meta-analysis may enhance the informed consent process for PMRT in breast cancer patients with 1-3 positive nodes.

\section{References}

1. Wenz F, Sperk E, Budach W, Dunst J, Feyer P, Fietkau R, Haase W, Harms W, Piroth MD, Sautter-Bihl ML, et al; Breast Cancer Expert Panel of the German Society of Radiation Oncology (DEGRO): DEGRO practical guidelines for radiotherapy of breast cancer IV: Radiotherapy following mastectomy for invasive breast cancer. Strahlenther Onkol 190: 705-714, 2014.

2. Dewis R and Gribbin J: Breast Cancer: Diagnosis and treatment. An Assessment of Need. National Institute for Health and Clinical Excellence Clinical Guidelines. National Collaborating Centre for Cancer, Cardiff (UK), February 2009.

3. EBCTCG (Early Breast Cancer Trialists' Collaborative Group), McGale P, Taylor C, Correa C, Cutter D, Duane F, Ewertz M, Gray R, Mannu G, Peto R, et al: Effect of radiotherapy after mastectomy and axillary surgery on 10-year recurrence and 20-year breast cancer mortality: Meta-analysis of individual patient data for 8135 women in 22 randomised trials. Lancet 383: 2127-2135, 2014 
4. Nordenskjöld AE, Fohlin H, Albertsson P, Arnesson LG, Chamalidou C, Einbeigi Z, Holmberg E, Nordenskjöld B and Karlsson P; Swedish Western and Southeastern Breast Cancer Groups: No clear effect of postoperative radiotherapy on survival of breast cancer patients with one to three positive nodes: A population-based study. Ann Oncol 26: 1149-1154, 2015.

5. Kong $M$ and Hong SE: Which patients might benefit from postmastectomy radiotherapy in breast cancer patients with T1-2 tumor and 1-3 axillary lymph nodes metastasis? Cancer Res Treat 45: 103-111, 2013.

6. Chitapanarux I, Tharavichitkul E, Jakrabhandu S, Klunklin P, Onchan W, Srikawin J, Pukanhaphan N, Traisathit P and Vongtama R: Real-world outcomes of postmastectomy radiotherapy in breast cancer patients with 1-3 positive lymph nodes: A retrospective study. J Radiat Res 55: 121-128, 2014.

7. Cosar R, Uzal C, Tokatli F, Denizli B, Saynak M, Turan N, Uzunoglu S, Ozen A, Sezer A, Ibis K, et al: Postmastectomy irradiation in breast in breast cancer patients with T1-2 and 1-3 positive axillary lymph nodes: Is there a role for radiation therapy? Radiat Oncol 6: 28, 2011.

8. Wu SG, He ZY, Li FY, Wang JJ, Guo J, Lin Q and Guan XX: The clinical value of adjuvant radiotherapy in patients with early stage breast cancer with 1 to 3 positive lymph nodes after mastectomy. Chin J Cancer 29: 668-676, 2010.

9. Ragaz J, Jackson SM, Le N, Plenderleith IH, Spinelli JJ, Basco VE, Wilson KS, Knowling MA, Coppin CM, Paradis M, et al: Adjuvant radiotherapy and chemotherapy in node-positive premenopausal women with breast cancer. N Engl J Med 337: 956-362, 1997.

10. Su YL, Li SH, Chen YY, Chen HC, Tang Y, Huang CH, Chou FF, Wu SC and Rau KM: Post-mastectomy radiotherapy benefits subgroups of breast cancer patients with T1-2 tumor and 1-3 axillary lymph node(s) metastasis. Radiol Oncol 48: 314-322, 2014.

11. Moo TA, McMillan R, Lee M, Stempel M, Patil S, Ho A and El-Tamer M: Selection criteria for postmastectomy radiotherapy in t1-t 2 tumors with 1 to 3 positive lymph nodes. Ann Surg Oncol 20: 3169-3174, 2013.

12. Huang CJ, Hou MF, Chuang HY, Lian SL, Huang MY, Chen FM, Fu OY and Lin SF: Comparison of clinical outcome of breast cancer patients with T1-2 tumor and one to three positive nodes with or without postmastectomy radiation therapy. Jpn J Clin Oncol 42: 711-720, 2012.

13. He ZY, Wu SG, Zhou J, Li FY, Lin Q, Lin HX and Sun JY: Postmastectomy radiotherapy improves disease-free survival of high risk of locoregional recurrence breast cancer patients with T1-2 and 1 to 3 positive nodes. PLoS One 10: e0119105, 2015.

14. Tendulkar RD, Rehman S, Shukla ME, Reddy CA, Moore H, Budd GT, Dietz J, Crowe JP and Macklis R: Impact of postmastectomy radiation on locoregional recurrence in breast cancer patients with 1-3 positive lymph nodes treated with modern systemic therapy. Int J Radiat Oncol Biol Phys 83: e577-e581, 2012
15. McBride A, Allen P, Woodward W, Kim M, Kuerer HM, Drinka EK, Sahin A, Strom EA, Buzdar A, Valero V, et al: Locoregional recurrence risk for patients with T1,2 breast cancer with 1-3 positive lymph nodes treated with mastectomy and systemic treatment. Int J Radiat Oncol Biol Phys 89: 392-398, 2014.

16. Harris EE, Freilich J, Lin HY, Chuong M and Acs G: The impact of the size of nodal metastases on recurrence risk in breast cancer patients with 1-3 positive axillary nodes after mastectomy. Int $\mathrm{J}$ Radiat Oncol Biol Phys 85: 609-614, 2013.

17. Overgaard M, Hansen PS, Overgaard J, Rose C, Andersson M, Bach F, Kjaer M, Gadeberg CC, Mouridsen HT, Jensen MB and Zedeler $\mathrm{K}$ : Postoperative radiotherapy in high-risk premenopausal women with breast cancer who receive adjuvant chemotherapy. Danish Breast Cancer Cooperative Group 82b Trial. N Engl J Med 337: 949-955, 1997.

18. Khan M, Gupta M and Seam R: Analysis of cardiac adverse events following postmastectomy hypofractionated radiotherapy. Chin Clin Oncol 3: 47, 2014.

19. Kunkler IH: Radiotherapy of the regional lymph nodes: Shooting at the sheriff? Breast 18 (Suppl 3): S112-S120, 2009.

20. Harris EE: Cardiac mortality and morbidity after breast cancer treatment. Cancer Control 15: 120-129, 2008

21. Doyle JJ, Neugut AI, Jacobson JS, Wang J, McBride R, Grann A, Grann VR and Hershman D: Radiation therapy, cardiac risk factors and cardiac toxicity in early-stage breast cancer patients. Int J Radiat Oncol Biol Phys 68: 82-93, 2007.

22. Berbers J, van Baardwijk A, Houben R, Heuts E, Smidt M, Keymeulen K, Bessems M, Tuinder S and Boersma LJ: 'Reconstruction: Before or after postmastectomy radiotherapy?' A systematic review of the literature. Eur J Cancer 50: 2752-2762, 2014.

23. Mitchell RE: Porcine acellular dermis-assisted breast reconstruction: Influence of adjuvant radiotherapy on complications and outcomes. Plast Reconstr Surg Glob Open 1: e77, 2013.

24. Rochlin DH, Jeong AR, Goldberg L, Harris T, Mohan K, Seal S, Canner J and Sacks JM: Postmastectomy radiation therapy and immediate autologous breast reconstruction: Integrating perspectives from surgical oncology, radiation oncology, and plastic and reconstructive surgery. J Surg Oncol 111: 251-257, 2015.

25. Kunkler IH, Canney P, van Tienhoven G and Russell NS; MRC/ EORTC (BIG 2-04) SUPREMO Trial Management Group: Elucidating the role of chest wall irradiation in 'intermediate-risk' breast cancer: The MRC/EORTC SUPREMO trial. Clin Oncol (R Coll Radiol) 20: 31-34, 2008. 